\title{
ON ABSOLUTELY SEGREGATED ALGEBRAS
}

\author{
MASATOSHII IKEDA
}

Cohomology groups of (associative) algebras have been introduced ( $\left.f_{0}\right):$ higher dimensions) and studied by G. Hochschild in his papers [2], [0] and [4]. 1-. 2-, and 3-dimensional cohomology groups are in closest connection with some classical properties of algebras. In particular, an algebra is absolutely segregated" if and only if its 2-dimensional cohomology groups are all trivial. It is thus of use and importance to determine the structure of algebras with universally vanishing 2-cohomology groups, i.e. absolutely segregated algebras; they form a class which is wider than the class of all algebras with universally vanishing 1-cohomology groups, i.e. separable algebras in the sense of the Dicl-son-IVedderburn theorem.

In the present note we offer a structural characterization of absolutely segregated algebras. As the preliminary we consider some simple lemmas on $M_{0}$-modules of an algebra (Definition 1 ) which have been studied by W. Gaschïtz $z^{2}$ in the case of finite groups and by H. Nagao, T. Nakayama. ${ }^{3)}$ and the writer ${ }^{1}$ in the case of algebras $(\$ 1)$. Combining these lemmas with a criterion for an algebra to have trivial $m$-dimensional cohomology groups, obtained by G. Hochschild in terms of Hochschild modules (Definition 3). we can refine Hochschild's criterion and show that the $m$-dimensional cohomology groups of an algebra are all trivial if and only if the same holds for $A_{k}$. Where $K$ is an extension of the ground field of $A(\S 2)$. Next, after showing that $A$ is absolutely segregated if and only if the basic algebra of $A$ is so $(\$ 3)$, we show a direct decomposition of the Hochschild module of the basic algebra of $A$ into two-sided modules ( $\S 4)$. Then, by the direct analysis of Hochschild modules, we have our structural characterization of absolutely segregated algebras ( $\$ 5)$.

The writer wishes to express his graticude to Professor T. Nakayama fo: his valuable suggestions.

\section{$\S 1$. $M_{0}$-modules of an algebra}

Let $A$ be, throughout this paper, an associative algebra with a finite ran': Received April 17, 1953.

1) An algebra $A$ is called absolutely segregated if any algebra $B$ containing a two-sided ideal $C$ such that $B / C \cong A$ contains a subalgcbra $A^{\prime}$ with $B=C+A^{\prime}$.

$\Rightarrow$ Gaschütz [1].

$\because$ Nagao and Nakayama [6].

: Ikeda [5]. 
over a field $F$. Moreover we assume, without mentioning each time, that $A$ has unir element 1 . Let

$$
A=\sum_{\kappa=1}^{n} \sum_{i=1}^{f(\kappa)} A e_{\kappa, i}=\sum_{\kappa=1}^{n} \sum_{i=1}^{f(\kappa)} e_{\kappa, i} A
$$

be direct decompositions of $A$ into indecomposable left or right ideals respectively. Here $e_{\kappa, i}$ are primitive idempotents such that $\sum_{\kappa=1}^{n} \sum_{i=1}^{f(\kappa)} e_{\kappa, i}=1$ and $A e_{\kappa, i}$ $\cong A e_{\lambda, j}\left(e_{\kappa}, i \cong e_{\lambda, j} A\right)$ if and only if $\kappa=\lambda$. For the sake of brevity, we write $e_{\kappa, 1}=e_{\kappa}$ for each $\kappa$. We use, moreover, matric units $c_{\kappa, i, j}$ with $c_{\kappa, i, j} c_{\lambda, h, k}$ $=\delta_{\kappa, \lambda} \delta_{j, h} c_{\kappa, i, k}, c_{\kappa, i, i}=e_{\mathrm{x}, i}$ for $\kappa, \lambda=1, \ldots, n ; i, j=1 . . f(\kappa)$ and $h, k=1, \ldots$, $f(\lambda)$.

Definition 1. Let $\mathfrak{M}$ be an $A$-module (one-sided or two-sided). $\mathfrak{M}$ is called an $M_{0}$-module if, for any $A$-module $\mathfrak{R}$ containing an $A$-submodule $\Re^{\prime}$ such that $\mathfrak{M} / \mathfrak{Y}^{\prime} \cong \mathfrak{M}$, there exists an $A$-subrnodule $\mathfrak{R}^{\prime \prime}$ of $\mathfrak{\Re}$ such that $\mathfrak{N}$ is the direct sum $\mathfrak{Y}=\mathfrak{\imath ^ { \prime }}+\mathfrak{\imath}^{\prime \prime}$.

Then we can easily verify

Lemma 1. Let $\mathfrak{M}$ be an A-left module. If $\mathfrak{M}=\mathfrak{M}_{1}+\mathfrak{M}_{2}$ is a direct decomposition of $\mathfrak{M}$ into A-left modules $\mathfrak{M}_{1}$ and $\mathfrak{M}_{2}$, then $\mathfrak{M}$ is an $M_{0}$-module if and only if $\mathfrak{M}_{1}$ and $\mathfrak{M}_{2}$ are $M_{0}$-modules.

Recently H, Nagao and T. Nakayama ${ }^{5)}$ proved

LeMma 2. If 1 asts as the identity operator on an A-left module $\mathfrak{M}$, then $\mathfrak{M}$ is an $M_{0}$-module if and only if $\mathfrak{M}$ is a restricted direct sum of A-submodules isomorphic to indecomposable left ideals $A e_{\kappa}$ of $A$.

By Lemma 2 we have

Leммa 3. If $\mathfrak{M}$ is an A-left module with finite rank over $F$ on which $I$

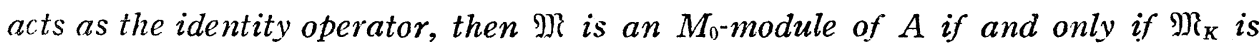
an $M_{i}$-module of $A_{K}$, where $K$ is an extension of $F$.

Proof. The "only if" part is trivial. We prove the "if" part. Assume that $\mathfrak{M}_{K}$ is an $M_{0}$-module of $A_{K}$. Then, by Lemma $2, \mathfrak{M}_{K}$ is a direct sum of finite number of $A_{K}$-submodules isomorphic to indecomposable left ideals of $A_{K}$, say $\mathfrak{l}_{K} \cong \sum_{i=1}^{r} \mathrm{~m}_{i}, \mathrm{~m}_{i} \cong A_{K} \widetilde{e}_{\kappa_{i}}$. Now, since $\widetilde{e}_{\kappa_{i}}$ is a primitive idempotent of $A_{K}$, we can assume that $A_{K} \widetilde{e}_{\kappa_{\ell}}$ appears as a direct component of $\left(A e_{\lambda}\right)_{K}$ for suitable $e_{\lambda}$. Since $\left(A e_{\lambda}\right)_{K}$ is a restricted direct sum of $A$-modules isomorphic to $A e_{\lambda}$, it is an $M_{v}$-module of $A$. Therefore, by Lemma 1 , its direct component $A_{K} \widetilde{e}_{\kappa_{i}}$ is also an $M_{0}$-module of $A$. Then, being the direct sum of submodules isomorphic to

5) Cf. Nagao and Nakayama [6]. 
$A_{K} \widetilde{e}_{\kappa_{i}}, \mathfrak{P}_{K}$ is an $M_{0}$-module of $\mathrm{A}$. Since $\mathfrak{M}_{K}$ is a direct sum of $\mathfrak{M}$ and a suitable $A$-submodule $\mathfrak{M}^{\prime}, \mathfrak{M}$ is an $\boldsymbol{M}_{0}$-module of $A$.

As for $A$-two-sided modules, ${ }^{6)}$ we can consider them as $A \times A^{\prime}$-left modules where $A^{\prime}$ is an algebra anti-isomorphic to $A$, and the above lemmas hold also for them.

\section{§2. Hnchschild modules and absolutely segregated algebras}

Now we turn to lemmas from the cohomology theory.7)

Definition 2. Let $\mathfrak{M}, \mathfrak{R}$ be $A$-two-sided modules. Then we call an $A$-twosided module $\mathfrak{R}$ an extension module of $\mathfrak{R}$ by $\mathfrak{M}$ if $\mathfrak{Z} \supset \mathfrak{N}$ and $\mathfrak{R} / \mathfrak{N} \cong \mathfrak{M}$. If a direct decomposition $\mathfrak{I}=\mathfrak{N}+\mathfrak{M}^{\prime}$ holds with an $A$-right submodule $\mathfrak{M}^{\prime}$, which is necessarily ( $A$-right) isomorphic to $\mathfrak{M}$, then we say that $\mathbb{Q}$ is a right inessential extension. If a direct decomposition $\mathfrak{L}=\mathfrak{l}+\mathfrak{M}^{\prime \prime}$ holds with an $A$-two-sided submodule $\mathfrak{R}^{\prime \prime}$, which is necessarily isomorphic to $\mathfrak{M}$, then we say the extension splits.

Lemma 4. (Hochschild) Let $\mathfrak{M}, \mathfrak{R}$ be A-two-sided modules. Then every right inessential extension of $\mathfrak{R}$ by $\mathfrak{M}$ splits if and only if $H^{(1)}(A ; R(\mathfrak{M}, \mathfrak{N}))=0$, where $R(\mathfrak{R}, \mathfrak{R})$ is an A-two-sided module consisting of right operator homomorphisins of $\mathfrak{M}$ into $\mathfrak{R}$ and the operation of an element a of $A$ on $R(\mathfrak{M}, \mathfrak{N})$ is defined by $(a * \lambda)(m)=a \lambda(m),(\lambda * a)(m)=\lambda(a m)(m \in \mathfrak{M}, \lambda \in R(\mathfrak{M}, \mathfrak{N}))$.

Definition 3. Let $P_{m}=A \otimes \ldots \otimes A$ be the $m$-fold direct product of the underlying vector space of $A$. We make $P_{m}$ into an $A$-two-sided module as follows: Let $A \in a_{0}, P_{m} \ni a_{1} \otimes \ldots \otimes a_{m}$. Then we define

$$
\begin{aligned}
\left(a_{1} \otimes \ldots \otimes a_{m}\right) * a_{0}= & a_{1} \otimes \ldots \otimes a_{m} a_{0} \text { and } \\
a_{0} *\left(a_{1} \otimes \ldots \otimes a_{m}\right)= & a_{0} a_{1} \otimes \ldots \otimes a_{m}-a_{0} \otimes a_{1} a_{2} \otimes \ldots \otimes a_{m}+\ldots \\
& \ldots+(-1)^{r} a_{0} \otimes \ldots \otimes a_{r} a_{r+1} \otimes \ldots \otimes a_{m}+\ldots \\
& +(-1)^{m-1} a_{0} \otimes a_{1} \otimes \ldots \otimes a_{m-1} a_{m} .
\end{aligned}
$$

We call $P_{m}$ thus defined the m-dimensional Hochschild module of $A$.

In distinction from ordinary direct products, we use the notation $\otimes$ for the Hochschild module $P_{m}$, while we use the notation $\times$ for ordinary direct products of two-sided modules, that is, $A^{(m)}=A_{1} \times \ldots \times A_{m}$ is an $A$-two-sided module under the operation $a_{0}\left(a_{1} \times \ldots \times a_{m}\right)=a_{0} a_{1} \times \ldots \times a_{m}$ and $\left(a_{1} \times \ldots\right.$ $\left.\times a_{m}\right) a_{0}=a_{1} \times \ldots \times a_{n_{0}} a_{0}$.

Lemma 5. (Hochschild) The m-dimensional cohomology groups of $A$ are all trivial if and only if every right inessential extension of any A-two-sided

6) " $A$-two-sided module" means " $A$-double module" ( $A$-Doppelmodul). Namely a module $\mathfrak{R}$ is an $A$-two-sided module if $\mathfrak{M}$ is an $A$-right as well as $A$-left module and satisfies $(a m) b$ $=a(m b) .(a, b \in A, m \in \mathbb{R})$.

i) Lemmas 4, 5 and 10 are in Hochschild [4]. 
module by $P_{m}$ splits.

Since $P_{m}$ is an $M_{1}$-module as an $A$-right module, every extension of any $A$-two-sided module by $P_{m}$ is right inessential. Therefore

LnMmA 6. The m-dimensional cohomology groups of $A$ are all trivial if and only if the m-dimensional Hochschild module $P_{m}$ of $A$ is an A-two-sided $M_{0^{-}}$ module.

Lemia 7. Let $\mathfrak{M}$ be an A-tu'o-sided module. If $\mathfrak{M}$ is an $M_{0}$-module as an A-right modile and if $1 \mathfrak{M}=0$, thien $\mathfrak{M}$ is an A-two-sided $M_{0}$-module.

Proof. Since every extension of any two-sided module $\Re$ by $\mathfrak{M}$ is right inessential, it is sufficient to show that $H^{(1)}(A ; R(\mathfrak{M}, \mathfrak{n}))=0$. From the def:nition, we have $(\lambda * a)(m)=\lambda(a m)=0$ for every $\lambda \in R(\mathfrak{M}, \mathfrak{M})$ and $m \in \mathfrak{M}$. Therefore $R(\mathfrak{M}, \mathfrak{l}) * A=0$. Let $\rho$ be a 1-cocycle from $A$ into $R(\mathfrak{M}, \mathfrak{l})$. Then $\delta \rho(a, b)=a * \rho(b)-\rho(a b)+\rho(a) * b=0$. Since $R(\mathfrak{M}, \mathfrak{N}) * A=0$, we have $a * \rho(b)$ $=\rho(a b)$. This shows that $\rho$ is an operator homomorphism of $A$ into $R(\mathfrak{M}, \mathfrak{\Re})$. Since $A$ has unit element $1, \rho(a)=a * \rho(1)=a * \rho(1)-\rho(1) * a=(\delta \rho(1))(a)$. Thus any 1-cocycle is a coboundary.

Since $P_{m}=1 * P_{m}+P_{m}^{(0)}$ where $P_{m}^{(0)}$ is the two-sided submodule of $P_{m}$ consisting of elements annihilated by 1 on the left-hand side, we have, by Lemmas 6 and 7 ,

Lemma 8. The m-dimensional cohomology groups of $A$ are all trivial if and only if $1 * P_{m}$ is an A-two-sided $M_{0}$-module, that is, $1 * P_{m}$ is isomorphis to a direct sum of indecomposable left ideals of $A \times A^{\prime}$.

On the other hand we have, from Lemmas 3 and 6 ,

Lemma 9. Let $\bar{K}$ be an extension of $F$. Then the m-dimensional cohomology groups of $A$ are all trivial if and only if the m-dimensional cohomology groups of $A_{K}$ are all trivial.

Definition 4. An algebra $A$ is called absolutely segregated if any algebra $B$ containing a two-sided ideal $C$ such that $B / C \cong A$ contains a subalgebra $A^{\prime}$ with $B=C+A^{\prime}$.

Then

Lenma 10. (Hochschild) An algebra $A$ is absolutely segregated if and only if the 2-dimensional cohomology groups of $A$ are all trivial.

By Lemmas 9 and 10, we have

Prorosition 1. An algebra $A$ is absolutely segregated if and only if $A_{K}$ is absolutely segregated, where $K$ is an extension of $F$. If $A$ is an algebra over an algebraic closed field, then $A$ is absolutely segregated if and only if $1 * P_{2}$ is isomorphic to a direct sum of A-two-sided modules isomorphic to the modules of the form $A e_{\kappa} \times e_{\lambda} A$. 
Now we give the next proposition which gives the relation between $1 * P_{m}$ and $A^{(m)}$.

Proposition 2.) By the correstondence $a_{1} \times \ldots \times a_{m} \rightarrow a_{1} *\left(a_{2} \gg \ldots\right.$. $A^{(m)}$ is mapped homomorplically onto $1 * P_{m-1}$ and the kernel of this homomorphism is isomorfinic to 1 : $P_{m}$.

Proof. The above mapping is obviously "onto." Since $\left(a_{0} a_{1}\right) *\left(a_{2}\right.$ ¿ $)$. . $\left.\left.\ldots \otimes a_{n n}\right)=a_{0} *\left(a_{2} \otimes \ldots \otimes a_{n}\right)\right)$ and $a_{1} *\left(a_{2} \ldots a_{m} a_{n+1}\right)=a_{1} *\left(\left(a_{2} \otimes \ldots\right.\right.$ ... $\left.\left.\otimes a_{m}\right) * a_{n+1}\right)=\left(a_{1} *\left(a_{2} \otimes ; \ldots a_{m}\right)\right) * a_{m+1}$, this is an $A$-homomorphism. Since $\left.1 *\left(a_{1} \otimes \ldots \otimes a_{n}\right)=a_{1} \otimes\right) \ldots \otimes a_{n}-1 \otimes\left(a_{1} *\left(a_{2} \otimes \ldots \otimes a_{m}\right)\right)$, the rest of the proposition is clear.

Remark. Since $a_{0} *\left(a_{1} \otimes \ldots a_{h t}\right)=a_{i} a_{1} \ldots \ldots a_{m}-a_{0} @\left(a_{1} *\left(a_{2} @ \ldots\right.\right.$ $\left.\otimes a_{m}\right)$ ), we see that the left multiplication of an element of $A$ to an element of $1 * P_{m}$ coincides with the ordinary multiplication.

\section{$\S 3$. The basic algebra of an absolutely segregated alisebra}

Definition 5. The subalgebra $A_{0}=E A E$ of $A$ is called the basic algehra of $A$, where $E=\sum_{\kappa=1}^{n} e_{\kappa}$.

Lemma 11. (Hochschild) $)^{9)}$ An algebra $A$ is absolutely serregrated if and only if any algebra $B$ containing a two-sided ideal $C$ such thai $B: C \cong A$ and $C^{2}=0$, contains a subalgebra $A^{\prime}$ sach tiuat $B=C+A^{\prime}$.

Proposition 3. An alsebra $A$ is absolutely segregated if and only if its basic algebra $A_{0}$ is absolutoly segrersated.

Proof. First we prove the "if" part. Assume that $A_{0}$ is absolutely segregated. Let $B$ be an algebra containing a two-sided ideal $C$ such that $B$; $C \cong A$. Then, by Lemma 11, we can assume $C^{2}=0$ and consequently we can construct matric units $\left\{\widetilde{c}_{\kappa, i, j}\right\}$ such that each $\widetilde{c}_{\kappa, i, j}$ belongs to the class $c_{\kappa, i, j} \bmod C$. Then $\left(\sum_{\kappa=1}^{n} \widetilde{c}_{\kappa, 1,1}\right) B\left(\sum_{\kappa=1}^{n} \widetilde{c}_{\kappa, 1,1}\right)=B_{0}$ contains $\left(\sum_{k=1}^{n} \widetilde{c}_{\kappa, 1,1}\right) C\left(\sum_{\kappa=1}^{n} \widetilde{c}_{\kappa, 1,1}\right)=C_{0}$ and $B_{0} / C_{0} \cong A_{0}$. Therefore $B_{0}$ contains a subalgebra $A_{0}^{\prime}$ such that $B_{0}=C_{0}+A_{\%}^{\prime}$. Since $A_{0}^{\prime} \cong A_{0}$. $A_{0}^{\prime}$ contains idempotents $\widetilde{c}_{\kappa}^{\prime}$ corresponding to $e_{\kappa}=c_{\kappa, 1,1}$ and, since $\sum_{\kappa=1}^{n} \widetilde{r}_{\kappa, 1,1}$ is the unit element of $B_{0}$, we have $\sum_{k=1}^{n} \widetilde{e}_{k}^{\prime}=\sum_{k=1}^{n} \widetilde{c}_{k, 1,1}$. Then $\widetilde{c}_{\kappa, l, i}(i \neq 1)$ and $\widetilde{e}_{k}^{\prime}$ forms mutually orthogonal primitive idempotents and therefore there exists matric units $\left\{\tilde{c}_{\kappa, i, j}^{\prime}\right\}$ such that $\tilde{c}_{\kappa, i, j}^{\prime}$ belongs to the class $c_{\kappa, i, j} \bmod C$ and $\tilde{c}_{\kappa, i, i}^{\prime}=\widetilde{c}_{\kappa, i, i}$ for $i \neq 1$ and $\tilde{c}_{\kappa, 1,1}^{\prime}=\widetilde{e}_{\kappa}^{\prime}$. Now we consider $A^{\prime}=\sum_{\kappa, \lambda, i, j} \tilde{c}_{\kappa, i, 1}^{\prime} A_{i}^{\prime} c_{\lambda, 1, j}^{\prime}$. It is clear that

8) Cf. Nakayama [7], Lemmas 4,1 and 4,2 .

9) Hochschild [2]. 
$A^{\prime}$ is a subalgebra and $B=C \cup A^{\prime}$. From $C_{0} \cap A_{0}^{\prime}=0$, it is clear that $C \cap A^{\prime}=0$. Thus $A$ is absolutely segregated.

Next we prove the "only if" part. Assume that $A$ is absolutely segregated and $B_{0}$ is an algebra containing a two-sided ideal $C_{0}$ such that $B_{0} / C_{0} \cong A_{0}$. Let $\left\{\widetilde{e}_{\kappa}\right\}$ be a system of idempotents in $B_{0}$ constructed in such a way that $\widetilde{e}_{\kappa}$ corresponds to $e_{\kappa}$ of $A_{0}$. Now let $\left\{\tilde{c}_{\kappa, i, j}\right\}(\kappa=1, \ldots, n ; i, j=1, \ldots, f(\kappa))$ be a system of symbols. $B_{0}=\sum_{\kappa, \lambda} \widetilde{e}_{\kappa} B_{\mathrm{c}} \widetilde{e}_{\lambda}+\sum_{\kappa} B_{0}^{(1)} \widetilde{e}_{\kappa}+\sum_{\kappa} \widetilde{e}_{\kappa} B_{0}^{(2)}+B_{0}^{(3)}$, where $B_{0}^{(1)}, B_{0}^{(2)}$ and $B_{0}^{(3)}$ consist of elements annihilated by left, right or two-sided multiplications of $\sum_{\kappa=1}^{n} \widetilde{e}_{\kappa}$, respectively. It is clear that $B_{0}^{(1)}, B_{0}^{(2)}$ and $B_{0}^{(3)}$ are contained in $C_{0}$. Let $B$ be the direct sum of modules $\widetilde{c}_{\kappa, i, 1} \widetilde{e}_{\kappa} B_{0} \widetilde{e}_{\lambda} \widetilde{c}_{\lambda, 1, j}, B_{0}^{(1)} \widetilde{e}_{\kappa} \widetilde{c}_{\lambda, 1, j}, \widetilde{c}_{\kappa, i, 1} \widetilde{e}_{\kappa} B_{0}^{(2)}$ and $B_{0}^{(3)}$ : $B=\sum_{\kappa, \lambda} \widetilde{c}_{k, i, j} \widetilde{e}_{\kappa} B_{0} \widetilde{e}_{\lambda} \widetilde{c}_{\lambda, 1, j}+\sum_{\kappa, i} B_{0}^{(1)} \widetilde{e}_{\kappa} \widetilde{c}_{\kappa, 1, i}+\sum_{\kappa, i} \widetilde{c}_{\kappa, i, 1} \widetilde{e}_{\kappa} B_{0}^{(2)}+B_{0}^{(3)}$. Now we set $\widetilde{c}_{\kappa, i}, j \widetilde{c}_{\lambda, h, k}$ $=\delta_{\kappa, \lambda} \delta_{j, h} \widetilde{c}_{\kappa, i, k}, \quad \widetilde{c}_{\kappa, 1,1}=\widetilde{e}_{\kappa}, \quad \widetilde{c}_{\kappa, i, j} B_{0}^{(1)}=0, B_{0}^{(2)} \widetilde{c}_{\kappa, i, j}=0$ and $\widetilde{c}_{k, i, j} B_{0}^{(3)}=B_{0}^{(3)} \widetilde{c}_{\kappa, i, j}=0$. Then it is clear that $B$ becomes an algebra. Let $C=\sum_{\kappa, \lambda, i, j} \widetilde{c}_{\kappa, i, 1} \widetilde{e}_{\kappa} C_{0} \widetilde{e}_{\lambda} \widetilde{c}_{\lambda, 1, j}$ $+\sum_{\kappa, i} B_{0}^{(1)} \widetilde{e}_{\kappa} \widetilde{c}_{\kappa, 1, i}+\sum_{\kappa, i} \widetilde{c}_{\kappa, i, 1} \widetilde{e}_{\kappa} B_{0}^{(2)}+B_{0}^{(2)}$, then $C$ is a two-sided ideal of $B$ and it is not hard to verify that $B / C \cong A$. Therefore $B$ contains a subalgebra $A^{\prime}$ such that $B=C+A^{\prime}$ and consequently $\left(\sum_{\kappa=1}^{n} \widetilde{e}_{\kappa}\right) B\left(\sum_{\kappa=1}^{n} \widetilde{e}_{k}\right)=\sum_{\kappa, \lambda} \widetilde{e}_{\kappa} B_{0} \widetilde{e}_{\lambda} \operatorname{contains}\left(\sum_{k=1}^{n} \widetilde{e}_{\kappa}\right) A^{\prime}\left(\sum_{k=1}^{n} \widetilde{e}_{\kappa}\right)$ $=A_{\nu}^{\prime}$ and $\sum_{\kappa, \lambda} \widetilde{e}_{\kappa} B_{0} \widetilde{e}_{\lambda}=A_{0}^{\prime}+\left(\sum_{\kappa=1}^{n} \widetilde{e}_{\kappa}\right) C\left(\sum_{\kappa=1}^{n} \widetilde{e}_{\kappa}\right)$. Since $B_{0}=\sum_{\kappa, \lambda} \widetilde{e}_{\kappa} B_{0} \widetilde{e}_{\lambda} \cup C_{0}$ and $A_{v}^{\prime} \cap C_{0}$ $=0$, we have $B_{0}=A_{0}^{\prime}+C_{0}$. This shows that $A_{0}$ is absolutely segregated.

\section{§4. A direct decomposition of $1 * P_{2}$ into two-sided submodules}

In this section we assume that $A$ is an algebra with rank $m$ over an algebraically closed field $\Omega$ and coincides with its basic algebra, i.e. satisfies the condition $(B)$ : if $A=\sum_{\kappa=1}^{n} A e_{\kappa}=\sum_{\kappa=1}^{n} e_{\kappa} A$ are direct decompositions into indecomposable left and right ideals of $A$, respectively, then $A e_{\kappa} \neq A e_{\lambda}\left(e_{\kappa} A \neq e_{\kappa} A\right)$ for $\kappa \neq \lambda$.

Lemma 12. $\left(1 * P_{2}: \Omega\right)=m^{2}-m$.

Proof. By Proposition 2, $A^{(2)} / \mathfrak{M} \cong 1 * P_{1} \cong A$ and $\mathfrak{M} \cong 1 * P_{2}$. Therefore $\left(1 * P_{2}: \Omega\right)=\left(A^{(2)}: \Omega\right)-(A: \Omega)=m^{2}-m$.

LEMma 13. Let $\left\{u_{i}(\kappa, \lambda)\right\}, \kappa \neq \lambda$, be an $\Omega$-basis of $e_{\kappa} A e_{\lambda}$, and let $\left\{u_{i}(\kappa, \kappa)\right\}$ be an $\Omega$-basis of $e_{\kappa} N e_{\kappa}$. Then, if we put $v_{i}(\kappa, \lambda)=e_{\kappa} \otimes u_{i}(\kappa, \lambda)-u_{i}(\kappa, \lambda) \otimes e_{\lambda}$, $A * v_{i}(\kappa, \lambda)$ and $v_{i}(\kappa, \lambda) * A$ are contained in $1 * P_{2}, A * v_{i}(\kappa . \lambda)$ is A-left-isomorpinic to $A e_{\kappa}$ and $v_{i}(\kappa, \lambda) * A$ is $A$-right-isomorphic to $e_{\lambda} A$. Moreover the sums $\bigcup_{\kappa, \lambda, i} A * v_{i}(\kappa$, $\lambda)$ and $\bigcup_{\kappa, \lambda, i} v_{i}(\kappa, \lambda) * A$ are direct.

Proof. Since $a * v_{i}(\kappa, \lambda)=a e_{\kappa} \otimes u_{i}(\kappa, \lambda)-a \otimes u_{i}(\kappa, \lambda)-a u_{i}(\kappa, \lambda) \otimes e_{\lambda}+a \otimes u_{i}(\kappa$, $\lambda)=a 2_{\kappa} \otimes u_{i}(\kappa, \lambda)-a u_{i}(\kappa, \lambda) \otimes e_{\lambda}, 1 * v_{i}(\kappa, \lambda)=v_{i}(\kappa, \lambda) \in 1 * P_{2}$. Therefore $A * v_{i}(\kappa$, 
$\therefore)$ and $v_{i}(\kappa, \lambda) * A$ are contained in $1 * P_{2}$. If $\sum_{\kappa, \lambda, i} a(\kappa, \lambda, i) * v_{i}(\kappa, \lambda)=0$ for some $a(\kappa, \lambda, i) \in A$, then $\left(\sum_{\kappa, \lambda, i} a(\kappa, \lambda, i) * v_{i}(\kappa, \lambda)=\right) 0=\sum_{\kappa, \lambda, i}^{\kappa, \lambda}\left(a(\kappa, \lambda, i) e_{\kappa} \otimes u_{i}(\kappa, \lambda)-a(\kappa, \lambda\right.$, i) $\left.u_{i}(\kappa, \lambda) \otimes e_{\lambda}\right)=\sum_{\kappa, \lambda, i} a(\kappa, \lambda, i) e_{\kappa} \otimes u_{i}(\kappa, \lambda)-\sum_{\lambda}\left(\sum_{\kappa, i} a(\kappa, \lambda, i) u_{i}(\kappa, \lambda)\right) \otimes e_{\lambda}$. Since $u_{i}(\kappa, \lambda)$ and $e_{\kappa}$ form an $\Omega$-basis of $A$, we have $a(\kappa, \lambda, i) e_{\kappa}=0$ and consequently $a(\kappa, \lambda, i) * v_{i}(\kappa, \lambda)=0$. This shows that the $\operatorname{sum} \bigcup_{\kappa, \lambda} A * v_{1}(\kappa, \lambda)$ is direct. At the same time, this shows that $A * v_{i}(\kappa, \lambda)=A e_{\kappa} * v_{i}(\kappa, \lambda) \cong A e_{\kappa}$. By the same way we have that the $\operatorname{sum}_{\kappa, \lambda, i} v_{i}(\kappa, \lambda) * A$ is direct and $v_{i}(\kappa, \lambda) * A \cong e_{\lambda} A$.

For the sake of brevity, we put $\left(A e_{\kappa}: \Omega\right)=s_{\kappa},\left(e_{\kappa} A: \Omega\right)=r_{\kappa}$ and $\left(e_{\kappa} A e_{\lambda}: \Omega\right)$ $=c_{\kappa}, \lambda$.

LEMMA 14. $1 * P_{2}=\sum_{\kappa \neq \lambda} A e_{\kappa} \otimes e_{\lambda} A+\sum_{\kappa, \lambda, i} A * v_{i}(\kappa, \lambda)=\sum_{\kappa \neq \lambda} A e_{\kappa} \otimes e_{\lambda} A+\sum_{\kappa, \lambda} v_{i}(\kappa, \lambda) * A$.

Proof. By direct computation, we see $A e_{\kappa} \otimes e_{\lambda} A \subset 1 * P_{2}$ if $\kappa \neq \lambda$. Since $P_{2}$ $=\sum_{\kappa, \lambda} A e_{\kappa} \otimes e_{\lambda} A$ and since $\sum_{\kappa} A e_{\kappa} \otimes e_{\kappa} A$ contains $\sum_{\kappa, \lambda, i} A * v_{i}(\kappa, \lambda)$ and $\sum_{\kappa, \lambda, i} v_{i}(\kappa, \lambda) * A$, the $\operatorname{sum}\left(\sum_{\kappa \neq \lambda} A e_{\kappa} \otimes e_{\lambda} A\right) \cup\left(\sum_{\kappa, \lambda, i} A * v_{i}(\kappa, \lambda)\right)$ and $\left(\sum_{\kappa \neq \lambda} A e_{\kappa} \otimes e_{\lambda} A\right) \cup\left(\sum_{\kappa, \lambda, i} v_{i}(\kappa, \lambda) * A\right)$ are direct. We show that these direct sums coincide with $1 * P_{2}$. To prove this, we compute the ranks of $\sum_{\kappa \neq \lambda} A e_{\kappa} \otimes e_{\lambda} A+\sum_{\kappa, \lambda, i} A v_{i}(\kappa, \lambda)$ and $\sum_{\kappa \neq \lambda} A e_{\kappa} \otimes e_{\lambda} A+\sum_{\kappa, \lambda, i} v_{i}(\kappa, \lambda) * A$. By Lemma 13 and the definition of $u_{i}(\kappa, \lambda),\left(\left(\sum_{\kappa \neq \lambda} A e_{\kappa}\left(\otimes e_{\lambda} A+\sum_{\kappa, \lambda} A * v_{i}(\kappa, \lambda)\right): \Omega\right)\right.$ $=\sum_{\kappa \neq=\lambda} s_{\kappa} r_{\lambda}+\sum_{\kappa, \lambda} s_{\kappa}\left(c_{\kappa, \lambda}-\delta_{\kappa, \lambda}\right)=\sum_{\kappa \neq \lambda} s_{\kappa} r_{\lambda}+\sum_{\kappa} s_{\kappa}\left(\sum_{\lambda} c_{\kappa, \lambda}-1\right)=\sum_{\kappa \neq \lambda} s_{\kappa} r_{\lambda}+\sum_{\kappa}^{\kappa, \lambda} s_{\kappa}\left(r_{\kappa}-1\right)=\sum_{\kappa, \lambda} s_{\kappa} r_{\lambda}$ $-\sum_{\kappa} s_{\kappa}=m^{2}-m=\left(1 * P_{2}: \Omega\right)$. In the same way, we have $\left(\left(\sum_{\kappa \neq \lambda} A e_{\kappa} \otimes e_{\lambda} A+\sum_{\kappa, \lambda, i} v_{i}(\kappa\right.\right.$, $\lambda) * A): \Omega)=\left(1 * P_{2}: \Omega\right)$.

LEMma 15. $\sum_{\kappa, \lambda, i} A * v_{i}(\kappa, \lambda)=\sum_{\kappa, \lambda, i} v_{i}(\kappa, \lambda) * A=\mathfrak{R}$ is a two-sided module.

Proof. Since $\sum_{\kappa, \lambda, i} A * v_{i}(\kappa, \lambda) \subset \sum_{\kappa, \lambda, i} A * v_{i}(\kappa, \lambda) * A \subset\left(\sum_{\kappa} A e_{\kappa} \otimes e_{\kappa} A\right) \cap 1 * P_{2}$, we have $1 * P_{2}=\sum_{\kappa, \lambda, i} A * v_{i}(\kappa, \lambda) * A+\sum_{\kappa \neq \lambda} A e_{\kappa} \otimes e_{\lambda} A$ and consequently $\sum_{\kappa, \lambda, i} A * v_{i}(\kappa, \lambda) * A=\sum_{\kappa, \lambda, i} A * v_{i}(\kappa$, 2.). By the same way, we have $\sum_{\kappa, \lambda, i} A * v_{i}(\kappa, \lambda) * A=\sum_{\kappa, \lambda, i}^{\kappa, \lambda, i} v_{i}(\kappa, \lambda) * A$.

By these two lemmas and the fact that $\sum_{\kappa \neq \lambda} A e_{\kappa} \otimes e_{\lambda} A \cong \sum_{\kappa \neq \lambda} A e_{\kappa} \times e_{\lambda} A, A$ is absolutely segregated if and only if $\mathfrak{M}$ is isomorphic to a direct sum of $A$-twosided modules $A e_{\kappa} \times e_{\lambda} A$.

LEMMA $16 \cdot e_{\kappa} a * v_{i}(\lambda, \nu)=\left(e_{\kappa} \otimes a u_{i}(\lambda, \nu)-e_{\kappa} a u_{1}(\lambda, \nu) \otimes e_{\nu}\right)-\left(e_{\kappa} \otimes e_{\kappa} a e_{\lambda}-e_{\kappa} a e_{\lambda}\right.$ $\left.\otimes e_{\lambda}\right) * u_{i}(\lambda, \nu)$.

\section{\$5 Structure of absolutely segregated algebras}

Consider an absolutely segregated algebra $A$ over an algebraically closed field $\Omega$ satisfying $(B)$. As was mentioned above, $\mathfrak{M}$ in Lemma 15 is a direct sum of submodules isomorphic to $A e_{\kappa} \times e_{\lambda} A$, say $\mathfrak{M} \cong \sum_{\kappa, \lambda} t_{\kappa, \lambda}\left(A e_{\kappa} \times e_{\lambda} A\right)$ as we want to write. 
Now we assume that the indices $1, \ldots, n$ are so arranged as $s_{1} \leqq \ldots \leqq s_{n}$. Then,

LEMMA 17. $s_{\lambda}=1+\sum_{\kappa} t_{\kappa, \lambda} s_{\kappa}$ and $r_{\kappa}=1+\sum_{\lambda} t_{\kappa, \lambda} r_{\lambda} . \quad t_{\kappa, \lambda}=0$ if $\kappa \geqslant \lambda$.

Proof. Since $\mathfrak{M}=\sum_{\kappa, \lambda, i} A * v_{i}(\kappa, \lambda)=\sum_{\kappa, \lambda, i} v_{i}(\kappa, \lambda) * A \cong \sum_{\kappa, \lambda} t_{\kappa, \lambda}\left(A e_{\kappa} \times e_{\lambda} A\right)$, we have, comparing indecomposable summands isomorphic to $e_{\lambda} A, \sum_{\kappa}\left(c_{\kappa, \lambda}-\delta_{\kappa, \lambda}\right)=s_{\lambda}-1$ $=\sum_{\kappa} t_{\kappa, \lambda} s_{\kappa}$. Since $s_{\lambda} \leqq s_{\kappa}$ for $\lambda \leqq \kappa, t_{\kappa, \lambda}=0$ if $\lambda \leqq \kappa$. By the same way, we have $r_{\kappa}=1+\sum_{\lambda} t_{\kappa, \lambda} r_{\lambda}$.

Corollary. $s_{1}=1$, that is, $A e_{1}=\Omega e_{1}$.

By this corollary, $\mathfrak{\Re}==\sum_{\lambda, i} A * v_{i}(1, \lambda)+\sum_{\kappa \neq 1 ; \lambda, i} A * v_{i}(\kappa, \lambda)=\sum_{\lambda, i} \Omega v_{i}(1, \lambda)+\sum_{\kappa \neq 1 ; \lambda, i} A * v_{i}(\kappa$, ג). We denote $\sum_{\kappa \neq 1} A * v_{i}(\kappa, \lambda)$ by $\mathfrak{M}_{1}$. On the other hand, $\mathfrak{M} \cong \cong \sum_{\kappa, \lambda} t_{\kappa, \lambda}\left(A \boldsymbol{e}_{\kappa} \times \boldsymbol{e}_{\lambda} A\right)$ and consequentiy $\mathfrak{M} \lambda=\sum_{\kappa, \lambda, i} \mathfrak{M}_{i}(\kappa, \lambda)$, where, for each pair $(\kappa, \lambda), \mathfrak{M}_{i}(\kappa, \lambda)$ are $t_{\kappa, \lambda}$ two-sided submodules of $\mathfrak{M}$ isomorphic to $A e_{\kappa} \times e_{\lambda} A$. Let $m_{i}(\kappa, \lambda)$ be the element of $\mathfrak{M}_{i}(\kappa, \lambda)$ corresponding to $e_{\kappa} \times e_{\lambda}$ by the above isomorphism, then $\mathfrak{M}_{i}(\kappa, \lambda)$ is generated by $m_{i}(\kappa, \lambda)$.

Lemma 18. $\mathfrak{M}_{1}=\sum_{\kappa \neq 1 ; \lambda, i} \mathfrak{M}_{i}(\kappa, \lambda)$; in particular, $\mathfrak{M}_{1}$ is a two-sided module.

Proof. Since $\mathfrak{M}=\sum_{\lambda, i} \mathscr{Q} v_{i}(1, \lambda)+\mathfrak{M}_{1}$, if $\kappa \neq 1, m_{i}(\kappa, \lambda) * a=e_{\kappa} * m_{i}(\kappa, \lambda) * a$ is contained in $e_{\kappa} \mathfrak{M}=e_{\kappa} \mathfrak{M}_{1}\left(\subset \mathfrak{M}_{1}\right)$ for any $a \in A$. Therefore $m_{i}(\kappa, \lambda) * A \subset \mathfrak{M}_{1}$ if $\kappa \neq 1$ and consequently $\mathfrak{M}_{1} \supseteq \sum_{\kappa \neq 1 ; \lambda, i} \sum_{i} * m_{i}(\kappa, \lambda) * A=\sum_{\kappa \neq 1, \lambda, i} \mathfrak{M}_{i}(\kappa, \lambda)$. On the other hand $\left(\sum_{\lambda, i} Q v_{i}(1, \lambda): \Omega\right)=\sum_{\lambda}\left(c_{1, \lambda}-\delta_{1, \lambda}\right)=r_{1}-1=\sum_{\kappa} t_{1, \kappa} r_{\kappa}=\left(\sum_{\kappa, i} \mathfrak{M}_{i}(1, \kappa): \Omega\right)=(\mathfrak{M}: \Omega)$ $-\left(\sum_{\kappa \neq 1, \lambda, i} \mathfrak{M}_{i}(\kappa, \lambda): \Omega\right)$. Therefore $\mathfrak{M}_{1}=\sum_{\kappa \neq 1 ; \lambda, i} \mathfrak{M}_{i}(\kappa, \lambda)$.

By Lemma 18, $\mathfrak{M} / \mathfrak{M}_{1} \cong \sum_{\kappa} t_{1, \kappa}\left(A e_{1} \times e_{\kappa} A\right)=\sum_{\kappa} t_{1, \kappa}\left(\Omega e_{1} \times e_{\kappa} A\right)$. Since $\mathfrak{M}$ $=\sum_{\lambda, i} \Omega v_{i}(1, \lambda)+\mathfrak{M}_{1}$, we can, for each $\kappa$, take $t_{1, \kappa}$ elements, say $x_{h}(1, \kappa)=\sum_{i} \omega_{i}(\kappa$, $h) v_{i}(1, \kappa)\left(\omega_{i}(\kappa, h) \in \Omega\right)$, as the representatives of the $t_{1, \kappa}$ classes corresponding to $t_{1, \kappa} e_{1} \times e_{\kappa}$ 's. Then, since $A e_{1}=\Omega e_{1}, \quad \mathfrak{N}=\sum_{\kappa, h} x_{h}(1, \kappa) * A+\mathfrak{M}_{1}$. We donote $\sum_{i} \omega_{i}(\kappa, h) u_{i}(1, \kappa)$ by $w_{h}(1, \kappa)$. Then $w_{h}(1, \kappa) \in e_{1} A e_{\kappa}$ and $x_{h}(1, \kappa)=e_{1} \otimes w_{h}(1, \kappa)$ $-w_{h}(1, \kappa) \otimes e_{\kappa}$.

Lemma 19. $e_{1} N=\sum_{\kappa, h} w_{h}(1, \kappa) A$ and $w_{h}(1, \kappa) A \cong e_{\kappa} A$ if $t_{1, \kappa} \neq 0$.

Proof. Assume that $\sum_{\kappa, h} w_{h}(1, \kappa) e_{\kappa} a_{\kappa, h}=0$ for some $a_{\kappa, h} \in A e_{2}$, where $\nu$ is an arbitrarily fixed. Since $t_{1,1}=0, \sum_{\kappa, h} w_{h}(1, \kappa) e_{\kappa} a_{\kappa, h}=\sum_{\kappa \neq 1 ; h} w_{h}(1, \kappa) e_{\kappa} a_{\kappa, h}=0$. Then $\sum_{\kappa \neq 1, h} x_{h}(1, \kappa) * e_{\kappa} a_{\kappa, h}=e_{1} \otimes\left(\sum_{\kappa \neq 1, h} w_{h}(1, \kappa) e_{\kappa} a_{\kappa, h}\right)-\sum_{\kappa \neq 1 ; h} w_{h}(1, \kappa) \otimes e_{\kappa} a_{\kappa, h}=-\sum_{\kappa \neq 1 ; h} w_{h}(1$, $\kappa) \otimes e_{\kappa} a_{\kappa, h}$. We can write $e_{\kappa} a_{\kappa, h}=e_{\kappa} a_{\kappa}, h e_{\nu}=\sum_{j} \beta(\kappa, h, j) u_{j}(\kappa, \nu)+\delta_{\kappa, \nu} \beta(h) e_{\nu}$, 
where $\beta(\ldots) \in \Omega$. Hence $-\sum_{\kappa \neq 1, h} w_{h}(1, \kappa) \otimes e_{\kappa} a_{\kappa, h}=-\sum_{\kappa \neq 1} \beta_{i} \beta(\kappa, h, j)\left(w_{h}(1, \kappa) \otimes u_{j} \backslash \kappa\right.$, $\nu))-\left(\sum_{h} \hat{\beta}(h) w_{h}(1, \nu)\right) \otimes e_{\nu}$. Now let $a(\kappa, j) \boldsymbol{e}_{\kappa}=-\sum_{h} \beta(\kappa, h, j) w_{h}(1, \kappa)$. Then $\mathfrak{M}_{1} \ni \sum_{\kappa \neq 1: j} a(\kappa, j) * v_{j}(\kappa, \nu)=\sum_{\kappa \neq 1: j} a(\kappa, j) e_{\kappa} \otimes u_{j}(\kappa, \nu)-\left(\sum_{\kappa \neq 1} a(\kappa, j) u_{j}(\kappa, \nu)\right) \otimes e^{\prime}$, $=-\sum_{\kappa \neq 1, h, j} \beta(\kappa, h, j)\left(w_{h}(1, \kappa) \otimes u_{j}(\kappa, \nu)\right)+\left(\sum_{\kappa \neq 1 ; h, j} \beta(\kappa, h, j) w_{h}(1, \kappa) u_{j}\left(h, l^{\prime}\right)\right) \otimes e_{\nu}$. Since $\sum_{\kappa \neq 1 ; h} w_{h}(1, \kappa) e_{\kappa} a_{\kappa, h}=\sum_{\kappa \neq 1 ; h, j} \beta(\kappa, h, j) w_{h}(1, \kappa) u_{j}(\kappa, \nu)+\sum_{h} \beta(h) w_{h}(1, \nu)=0$, $\sum_{\kappa \neq 1 ; h, j} \beta(\kappa, h, j) w_{h}(1, \kappa) u_{j}(\kappa, \nu)=-\sum_{h} \beta(h) w_{h}(1, \nu)$. Therefore $\sum_{\kappa \neq 1 ; 1, h} x_{h}(1, \kappa) * e_{\kappa} a_{\kappa, h}$ $=\sum_{\kappa \neq 1 ; j} a(\kappa, j) * v_{j}(\kappa, \nu) \in \mathfrak{M}_{1}$ and consequently $e_{\kappa} a_{\kappa}, h=0$.

Thus the sum $\bigcup_{\kappa, h} w_{h}(1, \kappa) A$ is direct. If $t_{1, \kappa} \neq 0$, then $u_{h}^{\prime}(1, \kappa) \neq 0$ and, as was shown above, $w_{h}(1, \kappa) A \cong e_{\kappa} A$. On the other hand $e_{1} N \supseteqq \sum_{\kappa, h_{h}} w_{h}(1, \kappa) A$ and $\left(e_{1} N: \Omega\right)$ $=r_{1}-1=\sum_{\kappa} t_{1, \kappa} r_{\kappa}=\left(\sum_{\kappa, h} w_{h}(1, \kappa) A: \Omega\right)$. Therefore $\epsilon_{1} N=\sum_{\kappa, h} w_{h}(1, \kappa) A$.

LEMMA 20. $\mathfrak{R}_{1}=\sum_{\kappa \neq 1 ; \lambda, i} A * v_{i}(\kappa, \lambda)=\sum_{\kappa \neq 1 ; \lambda, i} v_{i}(\kappa, \lambda) * A+\sum_{\kappa \neq 1} \sum_{\lambda, h, i} w_{h}(1, \kappa) * v_{i}(\kappa, \lambda) * A$.

Proof. By Lemma 19, we can take $w_{h}(1, \kappa)$ and $w_{h}(1, \kappa) u_{t}(\kappa, \lambda)(\kappa \neq 1)$ as an $Q$-basis of $e_{i} N$. By Lemma 16, $w_{h}\{1, \kappa) * v_{i}(\kappa, \lambda)=\left\{\boldsymbol{e}_{1}\left(w_{h}(1, \kappa) u_{i}(\kappa, \lambda)\right.\right.$ $\left.-w_{i n}(1, \kappa) u(\kappa, \lambda) \otimes e_{\lambda}\right)-x_{h}(1, \kappa) * u_{i}(\kappa, \lambda)$. Consequently, using the above $\Omega$ basis, we have $\sum_{\lambda, i} v_{i}(1, \lambda) * A=\sum_{\kappa \neq 1 ; 1} x_{i_{l}}(1, \kappa) * A+\sum_{\kappa \neq 1 ; h, i} w_{i}(1, \kappa) * v_{l}(\kappa, \lambda) * A$. Since $\mathfrak{M}=\sum_{\kappa \neq 1 ; \hbar} x_{h}(1, \kappa) * A+\mathfrak{M}_{1}$ and $\left(\sum_{\kappa \neq 1 ; \lambda, h, i}^{\kappa * 1 ; h} w_{h}(1, \kappa) * v_{i}(\kappa, \lambda) * A\right) \bigcup_{\kappa \neq 1 ; \lambda, i}\left(\sum_{i} v_{i}(\kappa, \lambda) * A\right) \subseteq \mathfrak{M} \mathfrak{R}_{1}$, we have $\mathfrak{M}_{1}=\left(\sum_{\kappa \neq 1} w_{\lambda, h, i}(1, \kappa) * v_{i}(\kappa, \lambda) * A\right) \cup\left(\sum_{\kappa \neq 1 ; \lambda, i} v_{i}(\kappa, \lambda) * A\right)$. It is easy to see that $\mathbb{M}_{1}=\sum_{\kappa \neq 1 ; \lambda, i} v_{i}(\kappa, \lambda) * A+\sum_{\kappa \neq 1} w_{h}(1, \kappa) * v_{i}(\kappa, \lambda) * A$.

Lemma 21. The following conditions (i), (ii) and (iii) hold for every $\kappa$.

(i) $\left.\mathfrak{M}_{\kappa}=\sum_{\mu>\kappa ; \lambda, i} A * v_{i}(\mu, \lambda)=\sum_{\mu>\kappa ; \lambda, i} \mathfrak{P}\right\}_{i}(\mu, \lambda)$.

(ii) There exist $t_{\kappa, \lambda}$ elements $w_{h}(\kappa, \lambda)$ in $e_{\kappa} A e_{\lambda}$ such that $e_{n} N=\sum_{\lambda, h} w_{h}(\kappa, \lambda) A$, $N e_{\kappa}=\sum_{\lambda=\kappa ; h} A w_{h}(\lambda, \kappa)$ and if $t_{\lambda, \kappa} \neq 0, A w_{h}(\lambda, \kappa)$ is A-left -isomorplic to $A e_{\lambda}$, and if $t_{\kappa, \lambda} \neq 0, w_{h}(\kappa, \lambda) A$ is A-right-isomorphic to $e_{\lambda} A$.

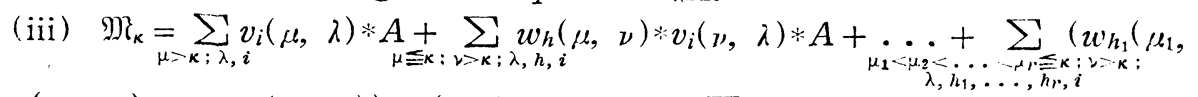
$\left.\left.\mu_{2}\right) w_{h_{2}}\left(\mu_{2}, \mu_{3}\right) \ldots w_{h_{r}}\left(\mu_{r}, \nu\right)\right) * v_{j}(\nu, \lambda) * A+\ldots \underset{\nu, \lambda_{, h_{1}, \ldots, h_{k}, i}}{ }\left(w_{h_{1}}(1,2) \ldots w_{h_{\kappa}}(\kappa, \nu)\right) * v_{i}(\nu$, $\lambda) * A$.

Proof. We assume that (i), (ii) and (iii) are satisfied for indices $\kappa \leqq p$. ( $p$ is a fixed integer.) We want to prove that (i), (ii) and (iii) hold for $\kappa=p$ +1 . From (ii), we can see, for $\kappa \leqslant p, s_{\kappa}=1+\sum_{\mu<\kappa}^{i} t_{\mu, \kappa}+\sum_{\mu_{1}<\mu_{2}<\kappa} t_{\mu_{1}, \mu_{2}} t_{\mu_{2}, \kappa}+\ldots$

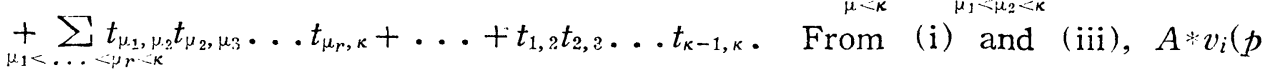
$+1, \lambda) \supseteqq \Omega v_{i}(p+1, \lambda)+\sum_{\mu \leqq \nu ; h} \Omega w_{h}(\mu, p+1) * v_{i}(p+1, \lambda)+\ldots+\sum_{\mu_{1}<\ldots<\mu_{r} \leqq \nu_{1} ; h_{1}, \ldots, h_{r}} \Omega\left(w_{h_{1}}\left(\mu_{1}, \mu_{2}\right) \ldots\right.$ 
$\left.\ldots w_{h_{r}}\left(\mu_{r}, p+1\right)\right) * v_{i}(p+1, \lambda)+\ldots+\sum_{h_{1}} \Omega\left(w_{h_{p}}\left(w_{h_{1}}(1,2) \ldots w_{h_{p}}(p, p+1)\right) * v_{i}(p\right.$ $+1, \lambda)$.

The rank of the right hand side is equal to $1+\sum_{\mu \geqq p} t_{\mu, p+1}+\ldots+\sum_{\mu_{1}<\mu_{2}}+\sum_{\mu_{\mu_{2}, \mu_{1}}} \ldots$ $t_{\mu r, p+1}+\ldots+t_{1,2} t_{3,2} \ldots t_{p, p+1}$. Since $s_{\kappa}=1+\sum_{\mu<\kappa} t_{\mu, \kappa}+\ldots+t_{1,2} t_{2,3} \ldots t_{\kappa-1, \kappa}$ for

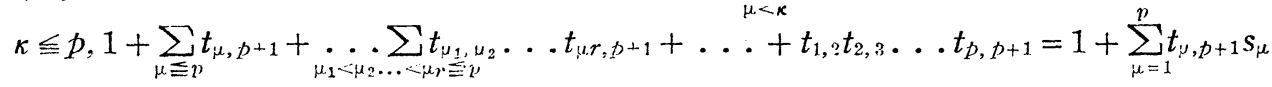
$=s_{i+1}=\left(A * v_{i}(p+1, \lambda): \Omega\right)$. This shows that $A * v_{i}(p+1, \lambda)=\Omega v_{i}(p+1, \lambda)$ $+\sum_{\mu=p ; h} \Omega w_{h}(\mu, p+1) * v_{i}(p+1, \lambda)+\ldots+\sum_{\mu_{1}<\ldots<\mu_{r}: p_{1} ; h_{1}, \ldots, h_{r}} \Omega\left(w_{h_{1}}\left(\mu_{1}, \mu_{2}\right) \ldots w_{h r}\left(\mu_{1}, p+1\right)\right) * v_{i}(p$ $+1, \lambda)+\ldots+\sum_{h_{1}, \ldots, h_{p}} 2\left(w_{h_{1}}(1,2) \ldots w_{h_{p}}(p, p+1)\right) * v_{i}(p+1, \lambda)$. Since $A * v_{i}(p+1, \lambda)$

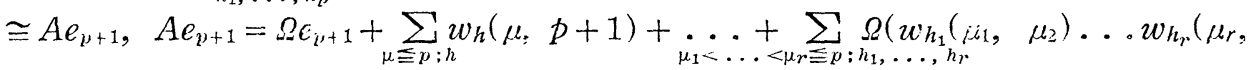
$p+1))+\ldots+\sum_{h_{1}}, \ldots, h_{r}\left(w_{h_{1}}(1,2) \ldots v v_{h_{p}}(p, p+1)\right)$. Then it is easy to see that $N e_{p+1}=\sum_{\kappa<p+1 ; h} A w_{h}(\kappa, p+1)$ and $A w_{h}(\kappa, p+1)=A e_{\kappa} w_{h}(\kappa, p+1) \cong A e_{\kappa}$. This proves the second part of (ii) for $\kappa=p+1$.

As was shown above, $\sum_{\lambda, i} A * v_{i}(p+1, \lambda)=\sum_{\lambda, i} \Omega v_{i}(p+1, \lambda)+\ldots+\sum_{\lambda, i, h_{1}, \ldots, h_{i}} w_{h_{1}}(1$, $\left.2),,, w_{h_{p}}(p, p+1)\right) * v_{i}(p+1, \lambda)$. Since $\mathfrak{M}_{p}=\sum_{\lambda, i} A * v_{i}(p+1, \lambda)+\mathfrak{M}_{p+1}$, we have $\mathfrak{M}_{p}=\left(\sum_{\lambda, i} \Omega v_{i}(p+1, \lambda)+\ldots+\sum_{\lambda, i, h_{1}, \ldots, h_{p}} \Omega\left(w_{h_{1}}(1,2) \ldots w_{h_{p}}(p, p+1)\right) * v_{i}(p+1, \lambda)\right)+\mathfrak{M}_{p+1}$. Then, by the same way used in Lemma 17 , we have $\mathfrak{M}_{p+1} \supseteq \sum_{\kappa+1} \mathfrak{M}_{i}(\kappa, \lambda)$. On the other hand, $\left(\sum_{\lambda, i} A * v_{i}(p+1, \lambda): \Omega\right)=s_{p+1}\left(\sum_{\lambda}\left(c_{p+1, \lambda}-\delta_{p+1, \lambda}\right)\right)=s_{p+1}\left(r_{p+1}-1\right)$ $=s_{p+1}\left(\sum_{\lambda} t_{p+1, \lambda} \boldsymbol{r}_{\lambda}\right)=\left(\sum_{\lambda, i} \mathfrak{M}_{i}(p+1, \lambda): \Omega\right)=\left(\mathfrak{M}_{p}: \Omega\right)-\left(\sum_{\kappa>p+1 ; \lambda, i} \mathfrak{M}_{i}(\kappa, \lambda)\right)$. Therefore $\mathfrak{M}_{i p+1}=\sum_{\kappa \rightarrow p+1 ; \lambda, i} \mathfrak{M}_{i}(\kappa, \lambda)$. This proves (i) for $\kappa=p+1$.

Now $\mathfrak{M}_{p} / \mathfrak{M}_{p+1} \cong \sum_{\lambda} t_{p+1, \lambda}\left(A e_{p+1} \times e_{\lambda} A\right)$. Since $\mathfrak{M}_{p}=\sum_{\lambda, i} \Omega v_{i}(p+1, \lambda)+\ldots$ $+\sum_{\lambda, i, h_{1}, \ldots, h_{p}} Q\left(w_{h_{1}}(1,2) \ldots w_{h_{p}}(p, p+1)\right) * v_{i}(p+1, \lambda)+\mathfrak{M}_{p+1}$, we can take $t_{p+1, \kappa}$ elements, say $x_{h}(p+1, \kappa)=\sum_{i} \omega_{i}(\kappa, h) v_{i}(p+1, \kappa)\left(\omega_{i}(\kappa, h) \in \Omega\right)$ as the representatives of the classes corresponding to $t_{p+1, \kappa} e_{p+1} \times e_{\kappa}$ 's. Then $\mathfrak{M}_{p}=\sum_{\kappa, h} A * x_{h}(p+1, \kappa) * A$ $+\mathfrak{M}_{p+1}$. As before, we denote $\sum_{i} \omega_{i}(\kappa, h) u_{i}(p+1, \kappa)$ by $w_{h}(p+1, \kappa)\left(\in e_{p+1} A e_{\kappa}\right)$. If $\sum_{\lambda, h} w_{h}(p+1, \lambda) e_{\lambda} a_{\lambda, h}=0$ for some $e_{\lambda} a_{\lambda, h} \in A$, then, since $t_{p+1, \lambda}=0$ for $\lambda \leqq p+1$, $\sum_{\lambda, h} w_{h}(p+1, \lambda) e_{\lambda} a_{\lambda, h}=\sum_{\lambda=1,1 ; h} w_{h}(p+1, \lambda) e_{\lambda} a_{\lambda, h}=0$ and consequently, by the same way used in Lemma 19, we have $\sum x_{h}(p+1, \lambda) * e_{\lambda} a_{\lambda, h} \equiv 0\left(\mathfrak{N}_{p+1}\right)$ which implies $\boldsymbol{e}_{\lambda} \boldsymbol{a}_{\lambda, h}=0$. This shows that $\boldsymbol{e}_{p+1} N \supseteqq \sum_{\lambda, h} w_{h}(p+1, \lambda) A$ and $w_{h}(p+1, \lambda) A \cong e_{\lambda} A$ if $t_{p+1, \lambda} \neq 0$. Comparing the ranks of $e_{p+1} N$ and $\sum_{\lambda, n} w_{h}(p+1, \lambda) A$, we have $e_{p+1} N$ $=\sum_{\lambda, h} w_{h}(p+1, \lambda) A$. This proves the first part of (ii) for $\kappa=p+1$.

Now we consider (iii). From the facts that $e_{p+1} N=\sum_{\kappa, h} w_{h}(p+1, \kappa) A$ and 
that $t_{p+1, \kappa}=0$ for $\kappa=1, \ldots, p+1$, we can take $w_{h}(p+1, \kappa)$ and $w_{h}(p+1, \kappa) u_{i}(\kappa$, 1) $(\kappa \neq 1, \ldots, p+1)$ as an $\Omega$-basis of $e_{p+1} N$. Using this $\Omega$-basis, we have $\boldsymbol{e}_{p+1} \otimes w_{h}(p+1, \kappa) u_{i}(\kappa, \lambda)-w_{h}(p+1, \kappa) u_{i}(\kappa, \lambda) \otimes e_{\lambda}=w_{h}(p+1, \kappa) * v_{i}(\kappa, \lambda)+x_{h}(p$ $+1, \kappa) * u_{i}(\kappa, \lambda)$. Consequently $\sum_{\kappa, i} v_{i}(p+1, \kappa) * A=\sum_{\kappa>p+1: h} x_{h}(p+1, \kappa) * A+\sum_{\kappa>p+1: \lambda, i, h} w_{h}(p$ $+1, \kappa) * v_{i}(\kappa, \lambda) * A$ and $\sum_{\kappa, i}\left(w_{h_{1}}\left(\mu_{1}, \mu_{2}\right) \ldots w_{h_{r}}\left(\iota_{r}, p+1\right)\right) * v_{i}(p+1, \kappa) * A$ $=\sum_{\kappa>p+1 ; h}\left(w_{h_{1}}\left(\mu_{1}, \mu_{2}\right) \ldots w_{h_{r}}\left(\mu_{i}, p+1\right)\right) * x_{h}(p+1, \kappa) * A+\sum_{\kappa=p+1 ;, i, i, h} w_{h_{1}}\left(\mu_{1}, \mu_{2}\right) \ldots w_{h_{r}}\left(\mu_{r}\right.$, $\left.p+1) w_{h}(p+1, \kappa)\right) * v_{i}(\kappa, \lambda) * A$. Then, by the facts that $\mathfrak{M}_{p}=\sum_{\kappa, n} A * x_{h}(p+1, \kappa) * A$

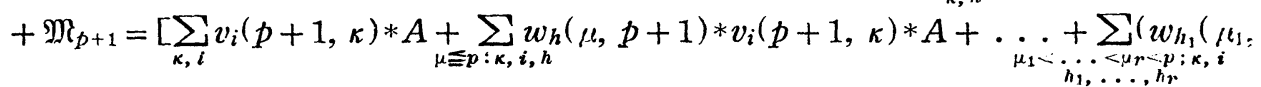
$\left.\left.\iota_{2}\right) . . w_{h_{r}}\left(\mu_{r}, p+1\right)\right) * v_{i}(p+1, \kappa) * A+\ldots{ }_{\kappa, i, h_{1}, \ldots, h_{\nu}}+\sum_{h_{1}}(1,2) \cdots w_{h_{\nu}}(p, p$ $\left.+1)) * v_{i}(p+1, \kappa) * A\right]+\left[\sum_{\kappa>p+1 ; \lambda, i} v_{i}(\kappa, \lambda) * \underset{\kappa>p+1 ; \mu_{1}<\ldots, \ldots, \mu_{r} \cong p ; \lambda, i}{A+w_{h_{1}}, \ldots, h_{r}}+\ldots \mu_{h_{r}}\left(\mu_{r}, \kappa\right)\right) * v_{i}(\kappa$, $\left.\lambda) * A+\underset{\kappa>p_{+1} ; \lambda, i, h_{1}, \ldots, h_{p}}{ }\left(w_{h_{1}}(1,2) \ldots w_{h_{\nu}}(p, \kappa)\right) * v_{i}(\kappa, \lambda) * A\right]$ and that $\mathfrak{M}_{p+1} \supseteqq\left[\sum_{\kappa>p+1 ; \lambda, i} v_{i}(\kappa\right.$, $\left.\left.\lambda) * A+\underset{\kappa>p+1 ; \lambda, i, h_{1}, \ldots, h_{p}}{\ldots}+\sum_{h_{1}}(1,2) \ldots w_{h_{p}}(p, \kappa)\right) * v_{i}(\kappa, \lambda) * A\right]+\left[\sum_{\kappa>p+1 ; \lambda, i, h} w_{k}(p+1, \kappa) * v_{i}(\kappa\right.$, $\left.\left.\lambda) * A+\underset{\kappa>p+1 ; \lambda, i, h_{1}, \ldots, h p_{+1}}{\ldots}+\ldots w_{h_{p}}(p, p+1) w_{h_{p+1}}(p+1, \kappa)\right) * v_{i}(\kappa, \lambda) * A\right]$, we have

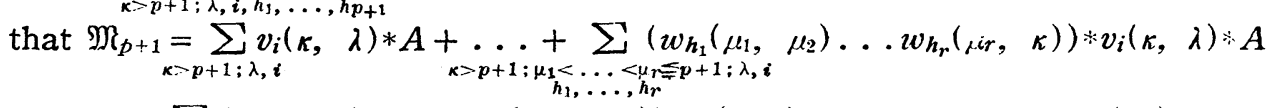
$+\ldots \ldots+\sum_{\kappa>p+1}\left(w_{h_{1}}(1,2) \ldots w_{h^{+1}}(p+1, \kappa)\right) * v_{i}(\kappa, \lambda) * A$. This proves (iii) for $\kappa$ $=p+1$. Therefore we have Lemma 21 by induction.

Proposition 4. Let $A$ be an absolutely segregated algebra over an algebraically closed fleld satisfying $(B)$, then there exists a system of non-negative integers $\left\{t_{\kappa, \lambda}\right\}$ such that $e_{\kappa} N \cong \sum_{\lambda} t_{\kappa, \lambda} e_{\lambda} A$ and $N e_{\kappa} \cong \sum_{\lambda} t_{\lambda, \kappa} A e_{\lambda}$ for each $\kappa$. Moreover $e_{\kappa} A e_{\kappa}$ $=\Omega e_{\kappa}$ for each $\kappa$.

Proof. As was shown above, we have that, for each $\kappa, N e_{\kappa}=\sum_{\lambda<\kappa} A w_{h}(\lambda, \kappa)$. Since $t_{\lambda, \kappa}=0$ for $\lambda>\kappa$ and $A w_{h}(\lambda, \kappa) \cong A e_{\lambda}$ if $t_{\lambda, \kappa} \neq 0$, we have $N e_{\kappa} \cong \sum_{\lambda} t_{\lambda, \kappa} A e_{\lambda}$. Then it can easily be seen that $N e_{\kappa}$ has only $\bar{A} \bar{e}_{\lambda}(\lambda<\kappa)$ as its composition residuemodules. This shows that $e_{\kappa} A e_{\kappa}=\Omega e_{\kappa}$. In the same way, we have $e_{\kappa} N$ $\cong \sum_{\lambda} t_{\kappa, \lambda} e_{\lambda} A$.

Now we consider a general algebra over an algebraically closed field, and prove

PROPOSITION 5. Let $A$ be an algebra over an algebraically closed field. Then $A$ is absolutely segregated if and only if there exists a system of non-negative integers $\left\{t_{\kappa, \lambda}\right\}$ such that $N e_{\kappa} \cong \sum_{\lambda} t_{\lambda, \kappa} A e_{\lambda}$, that is, $N$ is an A-left $M_{0}$-module.

Proof. By Proposition 2 and the fact that there exists such a system $\left\{t_{\kappa, \lambda}\right\}$ for $A$ if and only if the same holds for the basic algebra $A_{0}$ of $A$, it is sufficient to prove our assertion for an algebra satisfying $(B)$. 
As the "only if" part has been settled above, we prove the "if" part. As before we assume that $s_{1} \leqq \ldots \leqq s_{n}$. Then, by the above relation, we have that $s_{\kappa}-1=\sum_{\lambda} t_{\lambda, \kappa} s_{\lambda}$ and $t_{\lambda, \kappa}=0$ if $\lambda \gtrsim \kappa$. Therefore $N e_{\kappa} \cong \sum_{\lambda<\kappa} t_{\lambda, \kappa} A e_{\lambda}$. Now let $w_{h}(\lambda, \kappa)$ be $t_{\lambda, \kappa}$ elements corresponding to $e_{\lambda}$ by the above isomorphism. Then it is not hard to see that $e_{\kappa}, w_{h_{1}}\left(\kappa_{1}, \kappa_{2}\right), w_{h_{1}}\left(\kappa_{1}, \kappa_{2}\right) w_{h_{2}}\left(\kappa_{2}, \kappa_{3}\right), \ldots, w_{h_{1}}(1,2) w_{h_{2}}(2,3) \ldots$ $w_{h_{n-1}}(n-1, n)\left(\kappa_{i}=1, \ldots, n ; \kappa_{i}>\kappa_{i-1}\right)$ form an $\Omega$-basis of $A$. By this $\Omega$-basis we can decompose $\mathfrak{M}$ (of Lemma 15) into indecomposable left modules. Here, by Lemma 16, $e_{\kappa_{1}} \otimes w_{h_{1}}\left(\kappa_{1}, \kappa_{2}\right) w_{h_{2}}\left(\kappa_{2}, \kappa_{3}\right) \ldots w_{h_{r}}\left(\kappa_{r}, \kappa_{r+1}\right)-w_{h_{1}}\left(\kappa_{1}, \kappa_{2}\right) \ldots w_{h_{r}}\left(\kappa_{r}\right.$, $\left.\kappa_{r+1}\right) \otimes e_{\kappa_{r+1}}=\left(w_{h_{1}}\left(\kappa_{1}, \kappa_{2}\right) \ldots w_{h_{r-1}}\left(\kappa_{1-r}, \kappa_{r}\right)\right) *\left(e_{\kappa_{r}} \otimes w_{h_{r}}\left(\kappa_{r}, \kappa_{r+1}\right)-w_{h_{r}}\left(\kappa_{r}, \kappa_{r+1}\right)\right.$ $\left.(\otimes) e_{\kappa_{r+1}}\right)+\left(e_{\kappa_{1}} \otimes w_{h_{1}}\left(\kappa_{1}, \kappa_{2}\right) \ldots w_{h_{r-1}}\left(\kappa_{r-1}, \kappa_{r}\right)-w_{h_{1}}\left(\kappa_{1}, \kappa_{2}\right) \ldots w_{h_{r-1}}\left(\kappa_{r-1}, \kappa_{r}\right)\right.$ $\left.\otimes e_{\kappa_{r}}\right) * w_{h_{r}}\left(\kappa_{r}, \kappa_{r+1}\right)$. Therefore, by induction, we have $e_{\kappa_{1}} \otimes w_{h_{1}}\left(\kappa_{1}, \kappa_{2}\right) \ldots$ $w_{h_{r}}\left(\kappa_{r}, \kappa_{r+1}\right)-w_{h_{1}}\left(\kappa_{1}, \kappa_{2}\right) \ldots w_{h_{r}}\left(\kappa_{r}, \kappa_{r+1}\right) \otimes e_{\kappa_{r+1}}$ is contained $\operatorname{in}_{\kappa, \lambda, h} A *\left(e_{\kappa} \otimes w_{h}(\kappa, \lambda)\right.$ $\left.-w_{h}(\kappa, \lambda) \otimes e_{\lambda}\right) * A$. This shows that $\mathfrak{M}=\bigcup_{\kappa, \lambda, h} A *\left(e_{\kappa} \otimes w_{h}(\kappa, \lambda)-w_{h}(\kappa, \lambda) \otimes e_{\lambda}\right) * A$. On the other hand, $\left(A *\left(e_{\kappa} \otimes w_{h}(\kappa, \lambda)-w_{h}(\kappa, \lambda) \otimes e_{\lambda}\right) * A: \Omega\right) \leqq s_{\kappa} r_{\lambda}$ and consequently $\sum_{\kappa, \lambda, h}\left(A *\left(e_{\kappa} \otimes w_{h}(\kappa, \lambda)-w_{h}(\kappa, \lambda) \otimes e_{\lambda}\right) * A: \Omega\right) \leqq \sum_{\kappa, \lambda} t_{\kappa, \lambda} s_{\kappa} r_{\lambda}=\sum_{\lambda} r_{\lambda}\left(\sum_{\kappa} t_{\kappa}, \lambda s_{\kappa}\right)$ $=\sum_{\lambda} r_{\lambda}\left(s_{\lambda}-1\right)=\sum_{\kappa, \lambda} r_{\kappa} s_{\lambda}-\left(\sum_{\kappa \neq \lambda} A e_{\kappa} \otimes e_{\lambda} A: \Omega\right)-m=(\mathfrak{M}: \Omega)$. Therefore the sum $\bigcup_{\kappa, \lambda, h} A *\left(e_{\kappa} \otimes w_{h}(\kappa, \lambda)-w_{h}(\kappa, \lambda) \otimes e_{\lambda}\right) * A$ is direct and $A *\left(e_{\kappa} \otimes w_{h}(\kappa, \lambda)-w_{h}(\kappa, \lambda)\right.$ $\left(e_{\lambda}\right) * A \cong A e_{\kappa} \times e_{\lambda} A$. Thus $A$ is absolutely segregated.

TheOREM. Let $A$ be an algebra with unit element over a field $F$. Then $A$ is absolutely segregated if and only if

(i) $A / N$ is soparable,

(ii) the A-left-nsodule $N$ is directly decomposed into submodules isomorphic to some left-ideal direct components $A e_{\kappa}$ of $A$, i.e. there exists a system of nonnegative integers $\left\{t_{\kappa}, \lambda\right\}$ such that

$$
N e_{\kappa} \cong \sum_{\lambda} t_{\lambda, \kappa} A e_{\lambda}
$$

Proof. We prove the "if" part. Assume that $A$ satisfies (i) and (ii). Then from (ii), $N$ is an $A$-left $M_{0}$-module, therefore $N_{\Omega}(\Omega$ is an algebraic closer of $F$ ), the radical of $A_{\Omega}$, is also an $A_{\Omega}$-left $M_{0}$-module. Therefore $A_{\Omega}$ is absolutely segregated and consequently $A$ is absolutely segregated.

Next we prove the "only if" pari. Assume that $A$ is absolutely segregated and $A / N$ is inseparable. Then $(A / N)_{\Omega}$ contains a nilpotent element belonging to the centre of $(A / N)_{\Omega}$. Let $c$ be a representative of that class. Then $c$ belongs to the radical $N^{\prime}$ of $A_{\Omega}$ and there exists a primitive idempotent of $A$, say $e$, such that $c e \notin N_{\Omega}$. Since the residue class of $c \bmod N_{\Omega}$ is in the centre of $(A / N)_{\Omega}, e c e \neq 0$. Therefore $e A_{\Omega} e \supset e N^{\prime} e \neq 0$. This contradicts $e A_{\Omega} e=\Omega e$. Thus $A / N$ is separable, and $N_{\Omega}$ is an $A_{\Omega}$-left $M_{0}$-module. Hence $N$ is an $A$-left $M_{9}$-module. This completes the proof. 
Corollary. Let $A$ be an algebra without unit element, then $A$ is absolutely segregated if and only if $A^{*}=(1, A)$, the algebra obtained by adjunction of 1 to $A$, has the properties stated in our Theorem.

Added note. T. Nakayama and H. Nagao have given simpler proofs of our theorem. These will appear in this journal.

\section{REFERENCES}

[1] W. Gaschütz. Üter den Fundamentalsatz von Maschke zur Darstellungstheorie der endlichen Gruppen. Math. Z. Pd. 56 (1952).

[2] G. Hochschild. On the cohomology grours of an associative a!gebra, Ann. of Math., Vol. 46 (1945).

[3] - On the cohomology theory for associative algebras, Ann. of Math., 47 (1946).

[4] Cohomology and representatiors of associative algebras, Duke Math. J., Vol. 14 (1947).

[5] M. Ikeda. On a theorem of Gaschütz, Osaka Math. J. Vol. 5 (1953).

[6] H. Nagao and T. Nakayama. On the structure of $\left(M_{0}\right)$ - and $(M u)$-modules, fortheoming in Math. $Z$.

[7] T. Nakayama. Derivation and cohomology in simple and other rings I, Duke Math. J., Vol. 19 (1952). 